\title{
Hopf Bifurcation Analysis for the Model of the Chemostat with One Species of Organism
}

\author{
Haiyun Bai and Yanhui Zhai \\ School of Science, Tianjin Polytechnic University, Tianjin 300387, China \\ Correspondence should be addressed to Yanhui Zhai; y_h_zhai@yahoo.com.cn
}

Received 19 November 2012; Revised 3 March 2013; Accepted 3 March 2013

Academic Editor: Abdelaziz Rhandi

Copyright (C) 2013 H. Bai and Y. Zhai. This is an open access article distributed under the Creative Commons Attribution License, which permits unrestricted use, distribution, and reproduction in any medium, provided the original work is properly cited.

\begin{abstract}
We research the dynamics of the chemostat model with time delay. The conclusion confirms that a Hopf bifurcation occurs due to the existence of stability switches when the delay varies. By using the normal form theory and center manifold method, we derive the explicit formulas determining the stability and direction of bifurcating periodic solutions. Finally, some numerical simulations are given to illustrate the effectiveness of our results.
\end{abstract}

\section{Introduction}

Since late 60s, many researchers have been devoted to studying the chemostat, which is considered as an important laboratory set used for breeding microorganism and studying biological systems. In [1] Li et al. propose some new ideals by modifying the basic chemostat model with one species of organism and studying the Hopf bifurcations and stability of the modified one. In [2] $\mathrm{Li}$ et al. study the chemostat model with two time delays. They only research the stability of the equilibrium and the existence of the local Hopf bifurcation. However, some subtle mathematical questions on the behavior of solutions of the model are far from completely answered, for example, the bifurcating direction and stability of periodic solutions. Based on this, the main purpose of this study is to provide an insight into these unexplored aspects of the model by using the theory of the center manifold and the normal forms method.

Now we consider the basic model of the chemostat with one species of organism (see [3]):

$$
\begin{gathered}
\dot{x}(t)=-(d-p(y(t-\tau))) x(t), \\
\dot{y}(t)=\left(y_{0}-y(t)\right) d-\frac{1}{\gamma} p(y(t)) x(t),
\end{gathered}
$$

where $x(t)$ is the concentration of the organism at time $t$, $y(t)$ is the concentration of the nutrient at time $t, p(y)=$ $c y /(k+y)(c, k$ are positive constants) is the growing rate of $y$, $\gamma>0$ is the ratio of the mass of organism formed and the mass of substrate used, $y_{0}>0$ is the concentration of the input nutrient, $\tau>0$ is time lag of digestion, and $d>0$ is flowing rate.

\section{Stability and Local Hopf Bifurcation}

To consider the meaning of the biology, in the section we only focus on investigating the local stability of the interior equilibrium for the system (1). We know that if the equilibrium of system (1) is stable when $\tau=0$ and the characteristic equation of (1) has no purely imaginary roots for any $\tau>0$, it is also stable for any $\tau>0$. On the other hand, if the equilibrium of system (1) is stable when $\tau=0$ and there exist some positive values $\tau$ such that the characteristic equation of (1) has a pair of purely imaginary roots, there exists a domain concerning $\tau$ such that the equilibrium of system (1) is table in the domain.

When $y(t)<y_{0}$ for $\forall t>0$ and $c>d$, the system (1) has a unique interior equilibrium. We denote this unique interior equilibrium by $\left(x^{*}, y^{*}\right)$.

Then it satisfies

$$
\left(d-\frac{c y^{*}}{k+y^{*}}\right) x^{*}=0, \quad\left(y_{0}-y^{*}\right) d-\frac{c x^{*} y^{*}}{\gamma\left(k+y^{*}\right)}=0 .
$$


That is

$$
\left(x^{*}, y^{*}\right)=\left(\frac{\gamma c y_{0}-d y_{0}-d k}{c-d}, \frac{d k}{c-d}\right) .
$$

Let $x_{1}(t)=x(t)-x^{*}$ and $y_{1}(t)=y(t)-y^{*}$ and still denote $x(t), y(t)$, respectively.

Then system (1) becomes

$$
\begin{aligned}
& \dot{x}(t)=-d\left(x(t)+x^{*}\right)+\frac{c\left(y(t-\tau)+y^{*}\right)\left(x(t)+x^{*}\right)}{k+y(t-\tau)+y^{*}}, \\
& \dot{y}(t)=d\left(y_{0}-y(t)-y^{*}\right)-\frac{c\left(y(t)+y^{*}\right)\left(x(t)+x^{*}\right)}{\gamma\left(k+y(t)+y^{*}\right)} .
\end{aligned}
$$

The linearization of (4) around $\left(x^{*}, y^{*}\right)$ is

$$
\begin{aligned}
& \dot{x}(t)=(n-d) x(t)+m y(t-\tau), \\
& \dot{y}(t)=-\frac{n}{\gamma} x(t)-\left(\frac{m}{\gamma}+d\right) y(t),
\end{aligned}
$$

where $m=k c x^{*} /\left(k+y^{*}\right)^{2}$ and $n=c y^{*} /\left(k+y^{*}\right)$, whose characteristic equation is

$$
\lambda^{2}+\left(\frac{m}{\gamma}-n+2 d\right) \lambda-(n-d)\left(\frac{m}{\gamma}+d\right)+\frac{m n}{\gamma} e^{-\lambda \tau}=0 .
$$

By setting $(m / \gamma)+d=A, n-d=B$ and $(m n / \gamma)=K$, the characteristic equation (6) can be rewritten as

$$
\lambda^{2}+(A-B) \lambda-A B+K e^{-\lambda \tau}=0 .
$$

Lemma 1. When $\tau=0$ and

$$
A-B>0
$$

are met, the equilibrium $\left(x^{*}, y^{*}\right)$ of system (1) is asymptotically stable.

Proof. When $\tau=0$, (7) becomes

$$
\lambda^{2}+(A-B) \lambda-A B+K=0,
$$

whose characteristic value is

$$
\lambda_{1,2}=\frac{-(A-B) \pm \sqrt{(A-B)^{2}+4(A B-K)}}{2} .
$$

Obviously, when $(*)$ holds, the real parts of $\lambda_{1,2}$ are negative.

This completes the proof.

In the following, we investigate the distribution of the eigenvalues of the characteristic equation (7).

Lemma 2. Assume that

$$
K>A B
$$

is satisfied. Then (7) has a pair of purely imaginary roots $\pm i \omega_{0}$ when $\tau=\tau_{j}$, where

$$
\begin{gathered}
\omega_{0}=\left(\frac{-\left(A^{2}+B^{2}\right)+\sqrt{\left(A^{2}+B^{2}\right)^{2}-4\left(A^{2} B^{2}-K^{2}\right)}}{1 / 2}\right)^{1 / 2}, \\
\tau_{j}=\frac{1}{\omega_{0}}\left[\arccos \frac{\omega_{0}^{2}+A B}{K}+2 j \pi\right], \quad j=0,1,2, \ldots
\end{gathered}
$$

Proof. Let $i \omega(\omega>0)$ be a root of (7). Then

$$
-\omega^{2}+i(A-B) \omega-A B+K(\cos \omega \tau-i \sin \omega \tau)=0 .
$$

The separation of the real and imaginary parts yields

$$
\begin{gathered}
-\omega^{2}-A B+K \cos \omega \tau, \\
(A-B) \omega-K \sin \omega \tau .
\end{gathered}
$$

Hence

$$
\omega^{2}=\frac{-\left(A^{2}+B^{2}\right) \pm \sqrt{\left(A^{2}+B^{2}\right)^{2}-4\left(A^{2} B^{2}-K^{2}\right)}}{2} .
$$

Obviously, $(* *)$ implies that

$$
\omega_{0}=\left(\frac{-\left(A^{2}+B^{2}\right)+\sqrt{\left(A^{2}+B^{2}\right)^{2}-4\left(A^{2} B^{2}-K^{2}\right)}}{2}\right)^{1 / 2},
$$

and, hence,

$$
\tau_{0}=\frac{1}{\omega_{0}} \arccos \frac{\omega_{0}^{2}+A B}{K} .
$$

Define $\tau_{j}=\tau_{0}+\left(2 j \pi / \omega_{0}\right), j=0,1,2, \ldots$ Then $\left(\tau_{j}, \omega_{0}\right)$ solves (12).

This means that $i \omega_{0}$ is a root of (7) when $\tau=\tau_{j}, j=$ $0,1,2 \ldots$

This completes the proof.

Lemma 2 shows that there exist some positive values $\tau_{j}$ such that the characteristic equation (7) has a pair of purely imaginary roots.

Lemma 3. Let $\lambda(\tau)=a(\tau)+i b(\tau)$ be the root of (7) with $a\left(\tau_{j}\right)=0$ and $b\left(\tau_{j}\right)=\omega_{0}$. When $(* *)$ holds, $a^{\prime}\left(\tau_{j}\right)>0$.

Proof. By differentiating both sides of (7) with respect to $\tau$, we obtain

$$
2 \lambda \frac{d \lambda}{d \tau}+(A-B) \frac{d \lambda}{d \tau}-K e^{-\tau \lambda}\left(\lambda+\tau \frac{d \lambda}{d \tau}\right)=0 .
$$

Then

$$
\frac{d \lambda}{d \tau}=\frac{K \lambda e^{-\tau \lambda}}{2 \lambda+(A-B)-K \tau e^{-\tau \lambda}} .
$$


Substituting $\tau=\tau_{j}$ and $\lambda=i \omega_{0}$ into (17), we obtain

$$
\left.\frac{d \lambda}{d \tau}\right|_{\tau=\tau_{j}}=\frac{K i \omega_{0} e^{-\tau_{j} \omega_{0} i}}{2 i \omega_{0}+(A-B)-K \tau_{j} e^{-\tau_{j} \omega_{0} i}} .
$$

According to (12),

$$
\left.\frac{d \lambda}{d \tau}\right|_{\tau=\tau_{j}}=\frac{i \omega_{0}\left(\omega_{0}^{2}-i(A-B) \omega_{0}-A B\right)}{2 i \omega_{0}+(A-B)-\tau_{j}\left(\omega_{0}^{2}-i(A-B) \omega_{0}-A B\right)} .
$$

Hence

$$
\begin{aligned}
a^{\prime}\left(\tau_{j}\right) & =\left.\operatorname{Re} \frac{d \lambda}{d \tau}\right|_{\tau=\tau_{j}} \\
& =\frac{\omega_{0}^{2}\left(2 \omega_{0}^{2}+A^{2}+B^{2}\right)}{\left[A+B-\tau_{j} \omega_{0}^{2}+\tau_{j} A B\right]^{2}+\left[2 \omega_{0}+(A+B) \tau_{j} \omega_{0}\right]^{2}} \\
& >0 .
\end{aligned}
$$

The conclusion is completed.

Lemma 3 explains that the real parts $a^{\prime}(\tau)$ are monotonously increased in a small neighbourhood concerning $\tau_{j}$. In other words, the root of (7) crosses the imaginary axis from the left to the right as $\tau$ continuously varies from a number less than $\tau_{j}$ to one greater than $\tau_{j}$.

Lemma 4. When $(*)$ and $(* *)$ hold, then there exist $\tau_{0}<\tau_{1}<$ $\tau_{2}<\cdots$ such that all the roots of (7) have negative real parts when $\tau \in\left[0, \tau_{0}\right)$ and $(7)$ has at least one root with positive real parts when $\tau \in\left(\tau_{j}, \tau_{j+1}\right), j=1,2,3, \ldots$, where $\tau_{j}$ is defined as in (10).

In fact, according to Lemmas 1, 2, and 3, it is easy to obtain the results.

Applying Lemmas 2, 3, and 4 and the Hopf bifurcation theorem (see [4]), we have the following results.

Theorem 5. If $(*)$ and $(* *)$ are satisfied, then the equilibrium $\left(x^{*}, y^{*}\right)$ is asymptotically stable for $\tau \in\left[0, \tau_{0}\right)$ and unstable for $\tau>\tau_{0}$. System (4) undergoes a Hopf bifurcation at $\left(x^{*}, y^{*}\right)$ when $\tau=\tau_{j}, j=0,1,2 \ldots$, where $\tau_{j}$ is defined as in (10).

\section{Direction and Stability of the Bifurcating Periodic Solutions}

Throughout the following section, $C\left([-1,0] ; R_{+}^{2}\right)$ is a phase space, and $A$ stands for an operator, which is different from $A$ in Section 2.

In Section 3, we will research the stability and direction of the bifurcating periodic solutions of system (1). For convenience, let $t=s \tau$ and still denote $t$. Then the system (5) can be rewritten as

$$
\begin{aligned}
& \dot{x}(t)=\tau(n-d) x(t)+\tau m y(t-1), \\
& \dot{y}(t)=-\frac{\tau n}{\gamma} x(t)-\tau\left(\frac{m}{\gamma}+d\right) y(t) .
\end{aligned}
$$

By using the Taylor series and letting $\tau=\tau_{0}+\mu$, we have

$$
\begin{aligned}
\dot{x}(t)=\left(\tau_{0}+\mu\right)[ & (n-d) x(t)+m y(t-1) \\
& +\frac{k c}{\left(k+y^{*}\right)^{2}} x(t) y(t-1) \\
& \left.+O\left(\sqrt{x(t)^{2}+y(t-1)^{2}}\right)\right],
\end{aligned}
$$

$$
\begin{aligned}
\dot{y}(t)=\left(\tau_{0}+\mu\right)[ & -\frac{n}{\gamma} x(t)-\left(\frac{m}{\gamma}+d\right) y(t) \\
& +\frac{k c}{\gamma\left(k+y^{*}\right)^{2}} x(t) y(t) \\
& \left.+O \sqrt{x(t)^{2}+y(t)^{2}}\right] .
\end{aligned}
$$

Clearly, $\mu=0$ is the Hopf bifurcation value of system (21). Let $C_{1}=C\left([-1,0], R_{+}^{2}\right)$. For $\forall \phi=\left(\phi_{1}, \phi_{2}\right)^{T} \in C_{1}$, let

$$
L_{\mu}(\phi)=\left(\tau_{0}+\mu\right) A \phi(0)+\left(\tau_{0}+\mu\right) B \phi(-1),
$$

$f(\mu, \phi)$

$$
\begin{aligned}
= & \left(\tau_{0}+\mu\right) \\
& \times\left(\begin{array}{c}
\frac{k c}{\left(k+y^{*}\right)^{2}} \phi_{2}(-1) \phi_{1}(0)+O\left(\sqrt{\phi_{2}^{2}(-1)+\phi_{1}^{2}(0)}\right) \\
\frac{k c}{\gamma\left(k+y^{*}\right)^{2}} \phi_{1}(0) \phi_{2}(0)+O\left(\sqrt{\phi_{1}^{2}(0)+\phi_{2}^{2}(0)}\right)
\end{array}\right),
\end{aligned}
$$

where $A=\left(\begin{array}{cc}n-d & 0 \\ -(n / \gamma) & -((m / \gamma)+d)\end{array}\right), B=\left(\begin{array}{ll}0 & m \\ 0 & 0\end{array}\right)$.

By Riesz's representation theorem, there exists a matrix whose components are bounded variation functions $\eta(\theta, \mu)$ in $\theta \in[-1,0)$, such that

$$
L_{\mu}(\theta)=\int_{-1}^{0} d(\eta(\theta, \mu) \phi(\theta))
$$

for $\phi \in C$.

For $\phi \in C\left([-1,0], R^{2}\right)$, we define the operators $A$ and $R$ as

$$
\begin{gathered}
A(\mu) \phi(\theta)= \begin{cases}\frac{d \phi(\theta)}{d \theta}, & \theta \in[-1,0), \\
\int_{-1}^{0} d(\eta(t, \mu) \phi(t)), & \theta=0,\end{cases} \\
R(\mu) \phi(\theta)= \begin{cases}0, & \theta \in[-1,0), \\
f(\mu, \theta), & \theta=0 .\end{cases}
\end{gathered}
$$

Then the system (21) is equivalent to the following abstract differential equation [5]:

$$
\dot{u}_{t}=A(\mu) u_{t}+R(\mu) u_{t},
$$

where $u=\left(u_{1}, u_{2}\right)^{T}, u_{t}(\theta)=u(t+\theta)$ for $\theta \in[-1,0)$. 
As in [6], the bifurcating periodic solutions $x(t, \mu)$ of system (21) are indexed by a small parameter $\varepsilon$. A solution $x(t, \mu(\varepsilon))$ has amplitude $O(\varepsilon)$, period $T(\varepsilon)$, and nonzero Floquet exponent $\beta(\varepsilon)$ with $\beta(0)=0$. Under the present assumptions, $\mu, T$, and $\beta$ have expansions

$$
\begin{gathered}
\mu=\mu_{2} \varepsilon^{2}+\mu_{4} \varepsilon^{4}+\cdots, \\
T=\frac{2 \pi}{\omega}\left(1+T_{2} \varepsilon^{2}+T_{4} \varepsilon^{4}+\cdots\right), \\
\beta=\beta_{2} \varepsilon^{2}+\beta_{4} \varepsilon^{4}+\cdots .
\end{gathered}
$$

The sign of $\mu_{2}$ determines the direction of bifurcation: if $\mu_{2}>0(<0)$, then the Hopf bifurcation is forward (backward). $\beta_{2}$ determines the stability of the bifurcating periodic solutions: asymptotically orbitally stable (unstable) if $\beta_{2}<$ $0(>0)$. And $T_{2}$ determines the period of the bifurcating periodic solutions: the period increases (decreases) if $T_{2}>$ $0(<0)$.

Next, we only compute the coefficients $\mu_{2}, T_{2}, \beta_{2}$ in these expansions.

We define the adjoint operator $A^{*}$ of $A$ as

$$
A^{*} \psi(s)= \begin{cases}-\frac{d \psi(s)}{d s}, & s \in(0,1] \\ \int_{-1}^{0} d\left(\eta^{T}(t, 0) \psi(-t)\right), & s=0 .\end{cases}
$$

For $\phi \in C[-1,0]$ and $\psi \in C[0,1]$, define a bilinear form

$$
\langle\psi, \phi\rangle=\bar{\psi}^{T}(0) \phi(0)-\int_{-1}^{0} \int_{0}^{\theta} \bar{\psi}^{T}(\xi-\theta) d \eta(\theta) \phi(\xi) d \xi
$$

where $\eta(\theta)=\eta(\theta, 0)$.

To determine the normal form of operator $A$, we need to calculate the eigenvectors $q(\theta)$ and $q_{1}^{*}(s)$ of $A$ and $A^{*}$ corresponding to $i \tau_{0} \omega_{0}$ and $-i \tau_{0} \omega_{0}$, respectively.

Proposition 6. Assume that $q(\theta)$ and $q^{*}(s)$ are the eigenvectors of $A$ and $A^{*}$ corresponding to $i \tau_{0} \omega_{0}$ and $-i \tau_{0} \omega_{0}$, respectively, satisfying $\left\langle q^{*}, q\right\rangle=1$ and $\left\langle q^{*}, \bar{q}\right\rangle=0$.

Then

$$
\begin{gathered}
q(\theta)=(\alpha, \beta)^{T} e^{i \tau_{0} \omega_{0} \theta}=\left(i \omega_{0}+\frac{m}{\gamma}+d,-\frac{n}{\gamma}\right)^{T} e^{i \tau_{0} \omega_{0} \theta}, \\
q^{*}(s)=D\left(\alpha^{*}, \beta^{*}\right)^{T} e^{i \tau_{0} \omega_{0} s}=D\left(-\frac{n}{\gamma}, i \omega_{0}-n+d\right)^{T} e^{i \tau_{0} \omega_{0} s},
\end{gathered}
$$

where $\bar{D}=1 /\left((m / \gamma)+2 d-n-m \tau_{0} e^{-i \tau_{0} \omega_{0}}\right)$.

Proof. Without loss of generality, we just consider the eigenvector $q(\theta)$.

Firstly, when $\theta \in[-1,0)$, by the definition of $A$ and $q(\theta)$, we obtain the form $q(\theta)=(\alpha, \beta)^{T} e^{i \omega_{0} \tau_{0}}$ (here, $\alpha, \beta$ are unknown parameters).
In what follows, notice that $q(0)=(\alpha, \beta)^{T}$, and $A q(0)=$ $\int_{-1}^{0} d(\eta(t, \mu) \phi(t))=i \omega_{0} \tau_{0} q(0)$. We have

$$
\alpha=i \omega_{0}+\frac{m}{\gamma}+d, \quad \beta=-\frac{n}{\gamma} .
$$

Finally, by $\left\langle q^{*}, q\right\rangle=1$, we obtain the parameter $\bar{D}$.

The proof is completed.

Now we construct the coordinates of the center manifold $C_{0}$ at $\mu=0$.

Let

$$
z(t)=\left\langle q^{*}, u_{t}\right\rangle, \quad W(t, \theta)=u_{t}(\theta)-2 \operatorname{Re}\{z(t) q(\theta)\} .
$$

On the center manifold $C_{0}$, we have

$$
W(t, \theta)=W(z(t), \overline{z(t)}, \theta)
$$

where

$$
W(z, \bar{z}, \theta)=W_{20}(\theta) \frac{z^{2}}{2}+W_{11}(\theta) z \bar{z}+W_{02} \frac{\bar{z}^{2}}{2}+W_{30} \frac{z^{3}}{6} \cdots
$$

$z$ and $\bar{z}$ are local coordinates for the center manifold $C_{0}$ in the direction of $q$ and $q^{*}$, respectively. Since $\mu=0$, we have

$$
\begin{aligned}
z^{\prime}(t) & =i \tau_{0} \omega_{0} z(t)+\left\langle q^{*}(\theta), f(W+2 \operatorname{Re}\{z(t) q(\theta)\})\right\rangle \\
& =i \tau_{0} \omega_{0} z(t)+\overline{q^{*}(0)} f(W(z, \bar{z}, 0)+2 \operatorname{Re}\{z(t) q(0)\}) \\
& \triangleq i \tau_{0} \omega_{0} z(t)+\overline{q^{*}(0)} f_{0}(z, \bar{z})
\end{aligned}
$$

where

$$
f_{0}(z, \bar{z})=f_{z^{2}} \frac{z^{2}}{2}+f_{\bar{z}^{2}} \frac{\bar{z}^{2}}{2}+f_{z \bar{z}} z \bar{z}+\cdots
$$

We rewrite this as

$$
z^{\prime}(t)=i \tau_{0} \omega_{0} z+g(z, \bar{z})
$$

with

$$
\begin{aligned}
g(z, \bar{z}) & =\overline{q^{*}}(0) f_{0}(z, \bar{z}) \\
& =g_{20} \frac{z^{2}}{2}+g_{11} z \bar{z}+g_{02} \frac{\bar{z}^{2}}{2}+g_{21} \frac{z^{2} \bar{z}}{2}+\cdots
\end{aligned}
$$

By (27) and (36), we obtain

$$
\begin{aligned}
\dot{W} & =\dot{u}_{t}-\dot{z} q-\dot{\bar{z}} q \\
& = \begin{cases}A W-2 \operatorname{Re}\left\{\bar{q}^{*}(\theta) f_{0} q(\theta)\right\}, & \theta \in[-1,0) \\
A W-2 \operatorname{Re}\left\{\bar{q}^{*}(0) f_{0} q(0)\right\}+f_{0}, & \theta=0\end{cases} \\
& \triangleq A W+H(z, \bar{z}, \theta),
\end{aligned}
$$


where

$$
H(z, \bar{z}, \theta)=H_{20} \frac{z^{2}}{2}+H_{11} z \bar{z}+H_{02} \frac{\bar{z}^{2}}{2}+\cdots .
$$

By comparing the coefficients of the previous series, we obtain

$$
\begin{gathered}
\left(A-2 i \tau_{0} \omega_{0} I\right) W_{20}(\theta)=-H_{20}(\theta), \\
A W_{11}(\theta)=-H_{11}(\theta), \ldots
\end{gathered}
$$

Noticing $u_{t}=\left(u_{1 t}, u_{2 t}\right)=z q(\theta)+\bar{z} \overline{q(\theta)}+W(t, \theta)$, it follows that

$$
\begin{aligned}
y(t-1)= & \beta e^{-i \tau_{0} \omega_{0}} z+\bar{\beta} e^{i \tau_{0} \omega_{0}} \bar{z}+W_{20}^{(2)}(-1) \frac{z^{2}}{2} \\
& +W_{11}^{(2)}(-1) z \bar{z}+\cdots, \\
u_{1 t}(0)= & \alpha z+\bar{\alpha} \bar{z}+W_{20}^{(1)}(0) \frac{z^{2}}{2}+W_{11}^{(1)}(0) z \bar{z} \\
& +W_{02}^{(1)}(0) \frac{\bar{z}^{2}}{2}+\cdots, \\
u_{2 t}(0)= & \beta z+\bar{\beta} \bar{z}+W_{20}^{(2)}(0) \frac{z^{2}}{2}+W_{11}^{(2)}(0) z \bar{z} \\
& +W_{02}^{(2)}(0) \frac{\bar{z}^{2}}{2}+\cdots .
\end{aligned}
$$

Thus, from (39), we have

$$
\begin{gathered}
g_{20}=2 \bar{D} \frac{k c \tau_{0}}{\left(k+y^{*}\right)^{2}}\left(\alpha \alpha^{*} \beta e^{-i \tau_{0} \omega_{0}}+\frac{1}{\gamma} \alpha \alpha^{*} \beta\right), \\
g_{11}=\bar{D} \frac{k c \tau_{0}}{\left(k+y^{*}\right)^{2}}\left(\bar{\alpha} \alpha^{*} \beta e^{-i \tau_{0} \omega_{0}}+\alpha \alpha^{*} \bar{\beta} e^{i \tau_{0} \omega_{0}}\right. \\
\left.+\frac{1}{\gamma} \alpha \beta^{*} \bar{\beta}+\frac{1}{\gamma} \bar{\alpha} \beta \beta^{*}\right), \\
g_{02}=2 \bar{D} \frac{k c \tau_{0}}{\left(k+y^{*}\right)^{2}}\left(\bar{\alpha} \alpha^{*} \bar{\beta} e^{i \tau_{0} \omega_{0}}+\frac{1}{\gamma} \bar{\alpha} \bar{\beta} \beta^{*}\right), \\
+2 \bar{D} \frac{k c \tau_{0}}{\left(k+y^{*}\right)^{2}}\left(\alpha^{*} \beta e^{-i \tau_{0} \omega_{0}} W_{11}^{(1)}(0)+\alpha \alpha^{*} W_{11}^{(2)}(-1)\right. \\
+\alpha^{*} e^{i \tau_{0} \omega_{0}} W_{20}^{(1)}(0)+\frac{1}{2} \bar{\alpha} \alpha^{*} W_{20}^{(2)}(-1) \\
\left.+\frac{1}{\gamma} \alpha \beta^{*} W_{11}^{(2)}(0)\right)+\frac{1}{2 \gamma} \beta \beta^{*} W_{20}^{(1)}(0) \\
\gamma \beta \beta^{*} W_{11}^{(1)}(0)+\frac{1}{2 \gamma} \bar{\alpha} \beta^{*} W_{20}^{(2)}(0) .
\end{gathered}
$$

Since there are $W_{20}, W_{11}$ in $g_{21}$, we still need to compute them.

For $\theta \in[-1,0)$,

$$
\begin{aligned}
H(z, \bar{z}, \theta) & =-q^{*}(0) f_{0} q(\theta)-q^{*}(0) \bar{f}_{0} \bar{q}(\theta) \\
& =-g(z, \bar{z}) q(\theta)-g(z, \bar{z}) \bar{q}(\theta),
\end{aligned}
$$

and by comparing coefficients with (41),we obtain

$$
\begin{aligned}
H_{20}(\theta) & =-g_{20} q(\theta)-g_{02} \bar{q}(\theta), \\
H_{11}(\theta) & =-g_{11} q(\theta)-\bar{g}_{11} \bar{q}(\theta) .
\end{aligned}
$$

By substituting these relations into (42), we can derive the following equation:

$$
W_{20}^{\prime}(\theta)=2 i \tau_{0} \omega_{0} W_{20}(\theta)+g_{20} q(\theta)+\bar{g}_{02} \bar{q}(\theta) \text {. }
$$

By solving for $W_{20}(\theta), W_{11}(\theta)$, we obtain

$$
\begin{gathered}
W_{20}(\theta)=\frac{i g_{20}}{\tau_{0} \omega_{0}} q(0) e^{i \tau_{0} \omega_{0} \theta} \\
+\frac{i \bar{g}_{02}}{3 \tau_{0} \omega_{0}} \bar{q}(0) e^{-i \tau_{0} \omega_{0} \theta}+E_{1} e^{2 i \tau_{0} \omega_{0} \theta}, \\
W_{11}(\theta)=-\frac{i g_{11}}{\tau_{0} \omega_{0}} q(0) e^{i \tau_{0} \omega_{0} \theta}+\frac{i \bar{g}_{11}}{\tau_{0} \omega_{0}} \bar{q}(0) e^{-i \tau_{0} \omega_{0} \theta}+E_{2},
\end{gathered}
$$

where $E_{1}=\left(E_{1}^{(1)}, E_{1}^{(2)}\right), E_{2}=\left(E_{2}^{(1)}, E_{2}^{(2)}\right)$.

From the definition of $A$ and (46), we obtain

$$
\begin{aligned}
& \left(2 i \tau_{0} \omega_{0} I-\int_{-1}^{0} e^{2 i \tau_{0} \omega_{0} \theta} d \eta(\theta)\right) E_{1} \\
& =\frac{2 k c \tau_{0}}{\left(k+y^{*}\right)^{2}}\left(\alpha \beta e^{-i \omega_{0} \tau_{0}}, \frac{1}{\gamma} \alpha \beta\right)^{T} .
\end{aligned}
$$

Thus

$$
\begin{gathered}
E_{1}^{(1)}=\frac{2 k c \alpha \beta}{\left(k+y^{*}\right)^{2}}\left(e^{-i \omega_{0} \tau_{0}}\left(2 i \omega_{0}+\frac{m}{\gamma}+d\right)+\frac{m}{\gamma} e^{-2 i \omega_{0} \tau_{0}}\right) \\
\times\left(\left(2 i \omega_{0}-n+d\right)\left(2 i \omega_{0}+\frac{m}{\gamma}+d\right)\right. \\
\left.\quad+\frac{m n}{\gamma} e^{-2 i \omega_{0} \tau_{0}}\right)^{-1}, \\
E_{1}^{(2)}=\frac{2 k c \alpha \beta}{\left(k+y^{*}\right)^{2}}\left(2 i \omega_{0}-n+d-\frac{n}{\gamma} e^{-i \omega_{0} \tau_{0}}\right) \\
\times\left(\left(2 i \omega_{0}-n+d\right)\left(2 i \omega_{0}+\frac{m}{\gamma}+d\right)\right. \\
\left.\quad+\frac{m n}{\gamma} e^{-2 i \omega_{0} \tau_{0}}\right)^{-1} .
\end{gathered}
$$


Similarly, we have

$$
\begin{aligned}
E_{2}^{(1)}= & \frac{4 k c}{\left(k+y^{*}\right)^{2}}\left(\operatorname{Re}\left\{\bar{\alpha} \beta e^{-i \omega_{0} \tau_{0}}\right\}\left(\frac{m}{\gamma}+d\right)\right. \\
& \left.+\operatorname{Re}\{\bar{\alpha} \beta\} \frac{m}{\gamma} e^{-2 i \omega_{0} \tau_{0}}\right) \\
& \times\left((d-n)\left(\frac{m}{\gamma}+d\right)+\frac{m n}{\gamma} e^{-2 i \omega_{0} \tau_{0}}\right)^{-1}, \\
E_{2}^{(2)}= & \frac{4 k c}{\left(k+y^{*}\right)^{2}}\left(\frac{1}{\gamma}(n-d) \operatorname{Re}\{\bar{\alpha} \beta\}\right. \\
& \left.+\frac{n}{\gamma} \operatorname{Re}\left\{\bar{\alpha} \beta e^{-i \omega_{0} \tau_{0}}\right\}\right) \\
& \times\left((d-n)\left(\frac{m}{\gamma}+d\right)+\frac{m n}{\gamma} e^{-2 i \omega_{0} \tau_{0}}\right)^{-1} .
\end{aligned}
$$

Thus, we can compute the parameters $W(0)$ and $W(-1)$. In conclusion, we have computed all the coefficients in (39): $g_{20}, g_{11}, g_{02}$, and $g_{21}$.

Next, we can compute the following quantities:

$$
\begin{gathered}
C_{1}(0)=\frac{i}{2 \tau_{0} \omega_{0}}\left(g_{20} g_{11}-2\left|g_{11}\right|-\frac{1}{3}\left|g_{02}\right|^{2}\right)+\frac{g_{21}}{2}, \\
\mu_{2}=-\frac{\operatorname{Re}\left\{C_{1}(0)\right\}}{\operatorname{Re}\left\{\lambda^{\prime}\left(\tau_{0}\right)\right\}}, \\
\beta_{2}=2 \operatorname{Re}\left\{C_{1}(0)\right\}, \\
T_{2}=-\frac{\operatorname{Im}\left\{C_{1}(0)\right\}+\mu_{2}\left(\operatorname{Im}\left\{\lambda^{\prime}\left(\tau_{0}\right)\right\}\right)}{\omega_{0}} .
\end{gathered}
$$

From the discussion in Section 2, we know that $\operatorname{Re}\left\{\lambda^{\prime}\left(\tau_{0}\right)\right\}>0$. We therefore have the following result.

Theorem 7. If $\operatorname{Re}\left\{C_{1}(0)\right\}<0(>0)$, the direction of the Hopf bifurcation of the system (1) at the equilibrium $\left(x^{*}, y^{*}\right)$ when $\tau=\tau_{0}$ is forward (backward) and the bifurcating periodic solutions are orbitally asymptotically stable (unstable).

\section{Numerical Simulation}

In this section, we give a particular example to illustrate the effectiveness of our results. We take the coefficients $y_{0}=0.65$, $c=0.7, d=0.56, \gamma=2, k=0.1$ in (1). By simple computing, we have the equilibrium $\left(x^{*}, y^{*}\right)=(0.4,5.4)$, $\omega_{0} \doteq 0.2540, \tau_{0} \doteq 2.018$. Further, we obtain the numerical results directly by means of the software Matlab:

$$
\begin{gathered}
g_{20} \doteq-0.1839+0.0979 i, \quad g_{11} \doteq 0.0629+0.0164 i \\
g_{02} \doteq 0.0397-0.1067 i, \quad g_{21} \doteq-0.0271+0.0643 i . \\
\operatorname{Re}\left\{\lambda^{\prime}\left(\tau_{0}\right)\right\} \doteq 1.1591 \times 10^{-5}>0 . \\
\operatorname{Re}\left\{C_{1}(0)\right\} \doteq-0.0276<0 .
\end{gathered}
$$

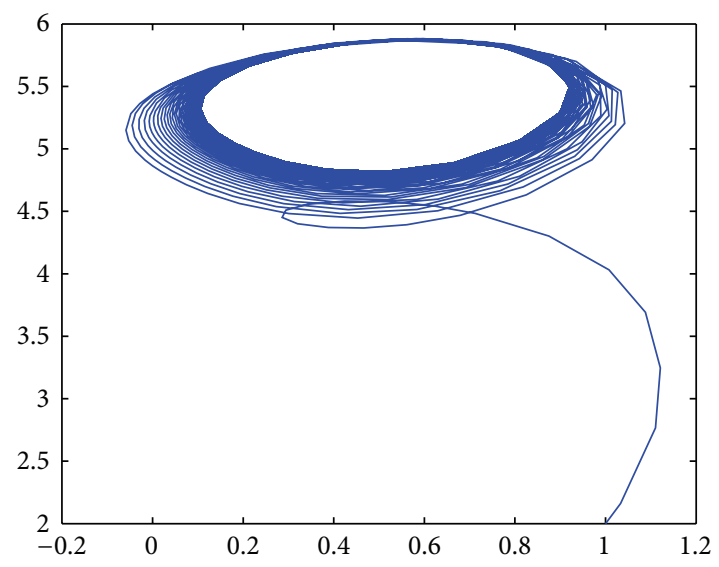

FIgURE 1: When $\tau=2.1$, the equilibrium $(0.4,5.4)$ is unstable and periodic solution occurs around them.

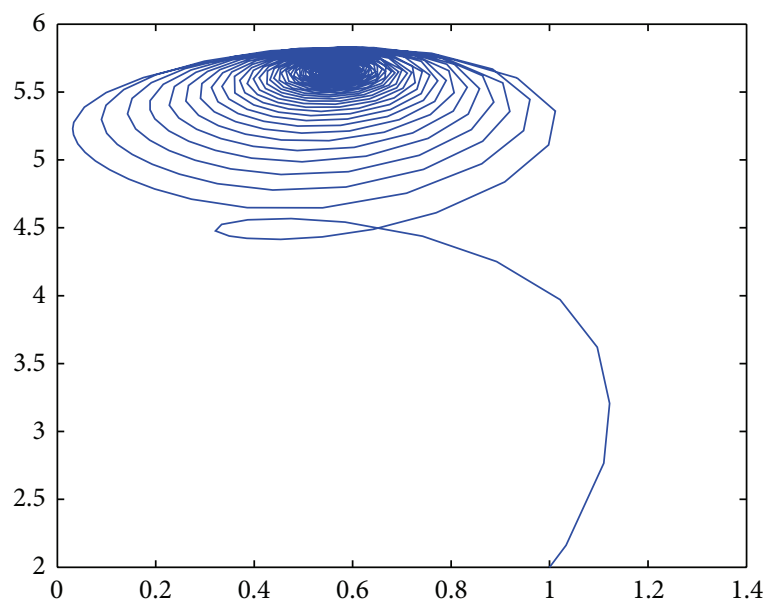

Figure 2: When $\tau=2$, the equilibrium $(0.4,5.4)$ becomes locally stable.

From the previous arithmetic, the equilibrium $(0.4,5.4)$ is asymptotically stable when $\tau=2<\tau_{0}=2.018$ (see Figure 2 ). When $\tau=2.1>\tau_{0}=2.018$ and $\operatorname{Re}\left\{C_{1}(0)\right\} \doteq-0.0276<0$, the stable periodic solutions occur from the equilibrium $(0.4,5.4)$ (see Figure 1).

Thus, the numerical simulation clarifies the effectiveness of our results.

\section{Conclusions}

In this paper, we have discussed the chemostat model with one species of organism. Firstly, we get the stable domain of equilibrium, and by regarding the delays $\tau$ as the bifurcation parameters and applying the theorem of Hopf bifurcation, we draw the sufficient conditions of the Hopf bifurcation. Further, by using the center manifold and the normal form method, we research the Hopf bifurcating direction and the stability of the model when $\tau=\tau_{0}$. Our analysis indicates that the dynamics of the model of the chemostat with one species of organism can be much more complicated than we may have 
expected. It is interesting to describe the global dynamics of the model by means of the local properties of the interior equilibrium.

\section{References}

[1] X. Li, J. Pan, and Q. Huang, "Hopf bifurcation analysis of some modified chemostat models," Northeastern Mathematical Journal, vol. 14, no. 4, pp. 392-400, 1998.

[2] X.-y. Li, M.-h. Qian, J.-p. Yang, and Q.-C. Huang, "Hopf bifurcations of a chemostat system with bi-parameters," Northeastern Mathematical Journal, vol. 20, no. 2, pp. 167-174, 2004.

[3] H. I. Freedman, J. W.-H. So, and P. Waltman, "Coexistence in a model of competition in the chemostat incorporating discrete delays," SIAM Journal on Applied Mathematics, vol. 49, no. 3, pp. 859-870, 1989.

[4] J. Hale, Theory of Functional Differential Equations, Springer, 1977.

[5] J. K. Hale and S. M. V. Lunel, Introduction to Functional Differential Equations, Springer, 1995.

[6] G. Mircea, M. Neamtu, and D. Opris, Dynamical Systems from Economy, Mechanic and Biology Described by Differential Equations with Time Delay, Mirton, 2003.

[7] J. Wei and C. Yu, "Hopf bifurcation analysis in a model of oscillatory gene expression with delay," Proceedings of the Royal Society of Edinburgh A, vol. 139, no. 4, pp. 879-895, 2009.

[8] N. A. M. Monk, "Oscillatory expression of Hes1, p53, and NF$\kappa \mathrm{B}$ driven by transcriptional time delays," Current Biology, vol. 13, no. 16, pp. 1409-1413, 2003.

[9] Y. Song and J. Wei, "Bifurcation analysis for Chen's system with delayed feedback and its application to control of chaos," Chaos, Solitons \& Fractals, vol. 22, no. 1, pp. 75-91, 2004.

[10] Y. Song, J. Wei, and M. Han, "Local and global Hopf bifurcation in a delayed hematopoiesis model," International Journal of Bifurcation and Chaos in Applied Sciences and Engineering, vol. 14, no. 11, pp. 3909-3919, 2004.

[11] S. Ruan and J. Wei, "On the zeros of transcendental functions with applications to stability of delay differential equations with two delays," Dynamics of Continuous, Discrete \& Impulsive Systems A, vol. 10, no. 6, pp. 863-874, 2003.

[12] J. Wei, "Bifurcation analysis in a scalar delay differential equation," Nonlinearity, vol. 20, no. 11, pp. 2483-2498, 2007. 


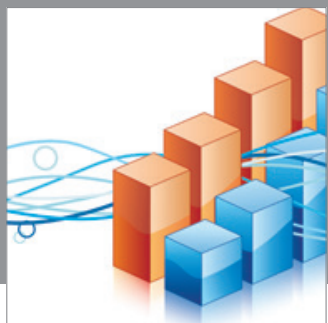

Advances in

Operations Research

mansans

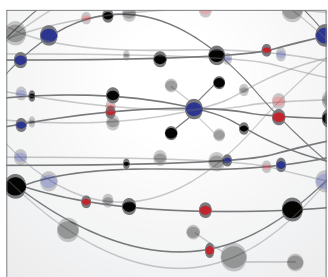

The Scientific World Journal
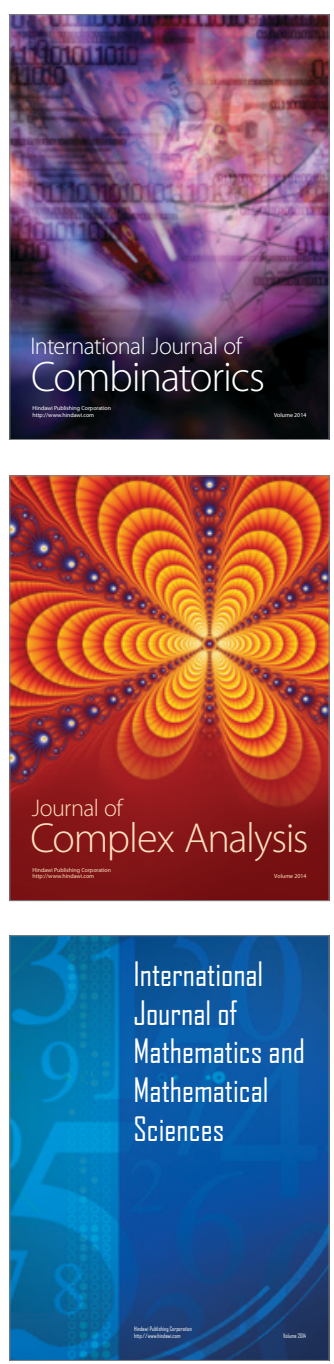
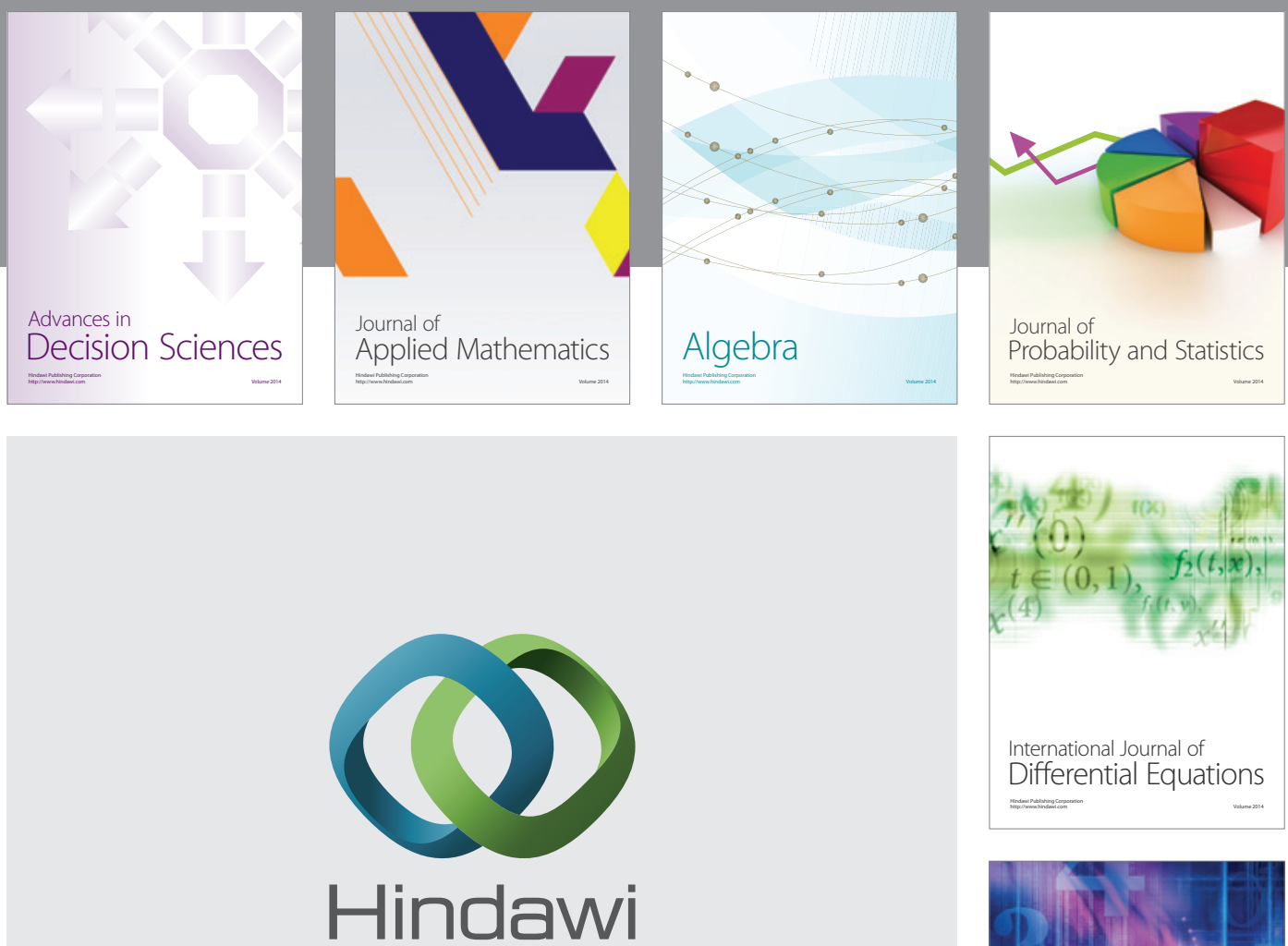

Submit your manuscripts at http://www.hindawi.com
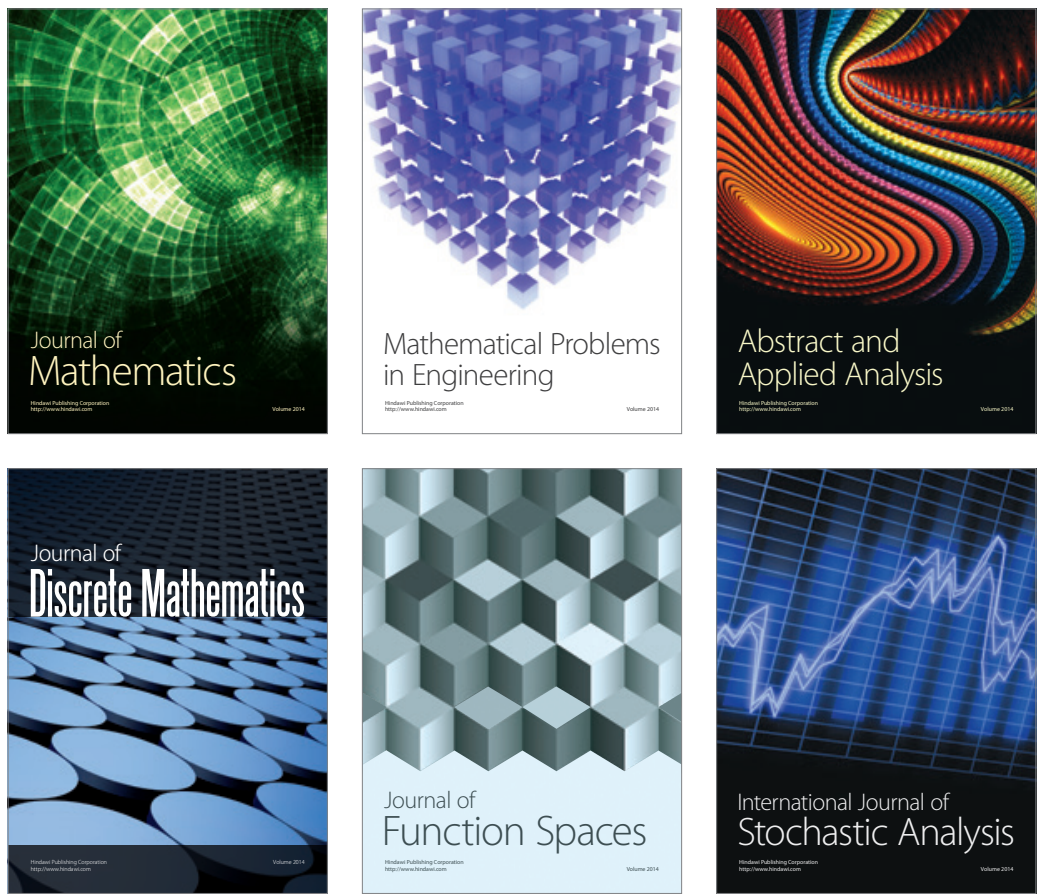

Journal of

Function Spaces

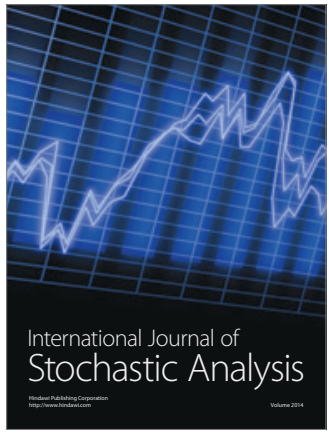

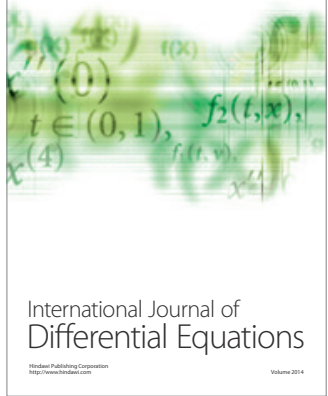
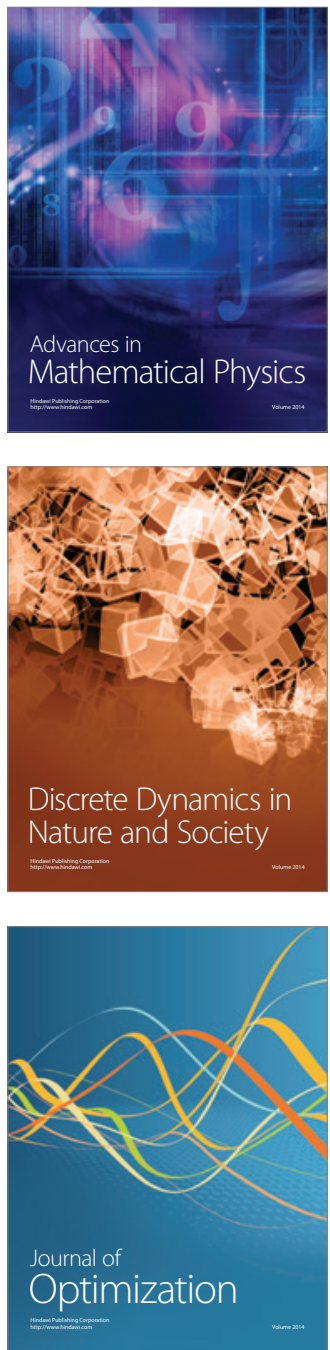